\title{
Origen anómalo de la arteria descendente anterior
}

\author{
Anomalous origin of the anterior descending artery \\ Carlos A. Martínez-Cano*, Sergio A. Romero-Seguro, Cristhian F. Ramírez-Ramos, \\ Diego A. Echeverry y Luis A. Bolívar-Mejía \\ Departamento de Cardiología Clínica, Clínica CardioVID, Medellín, Colombia
}

\section{Resumen}

Se considera origen anómalo de una arteria coronaria cuando esta no se origina de su seno de Valsalva respectivo. La prevalencia estimada varía entre el $0.6 \%$ y el $1.3 \%$. Si bien la mayoría estarán asintomáticos, el $20 \%$ puede debutar con arritmias, síncope, infarto del miocardio o muerte súbita. Se expone el caso de una mujer de 42 años, con historia de hipertensión arterial, quien, durante una prueba de esfuerzo para prescripción de ejercicio, presentó bloqueo de rama izquierda. Ante la ausencia de síntomas se decidió realizar una angiografía coronaria por tomografía, la cual mostró un origen anómalo de la arteria descendente anterior desde el seno de Valsalva derecho. Se consideró realizar angiografía coronaria para evaluar la compresión dinámica durante el ciclo cardiaco; sin embargo, la paciente no aceptó intervenciones adicionales, por lo que se indicó restricción del ejercicio, metoprolol y seguimiento. A la fecha no reporta complicaciones. Las anomalías de las arterias coronarias son defectos cardiacos poco comunes, y entre estos, las anormalidades de la arteria descendente anterior son aún menos habituales. Son una causa frecuente de muerte súbita en atletas. Sus características anatómicas y la presencia de síntomas ayudarán a elegir a los pacientes que se beneficiarán del manejo quirúrgico.

Palabras clave: Anomalías congénitas. Enfermedad de la arteria coronaria. Muerte súbita.

\section{Abstract}

An anomalous origin of a coronary artery is considered when it does not originate from its respective Valsalva sinus. The estimated prevalence varies between $0.6 \%$ to $1.3 \%$. While the majority will be asymptomatic, $20 \%$ may debut with arrhythmias, syncope, myocardial infarction or sudden death. It is presented a case of a 42 year old woman, with a history of high blood pressure. During exercise stress test for exercise prescription she presented a left bundle branch block. In absence of symptoms, it was decided to perform a coronary angiotomography that showed an anomalous origin of the anterior descending artery from the right Valsalva sinus. It was considered to perform coronary angiography to evaluate dynamic compression during the cardiac cycle, however, the patient did not accept additional interventions, so exercise restriction was indicated, metoprolol as drug treatment and follow up, not report complications to date. Coronary artery abnormalities are rare heart defects and within these abnormalities of the anterior descending artery are even less frequent. They are a frequent cause of sudden death in athletes. Its anatomical characteristics as the presence of symptoms will help to choose the patients who will benefit from surgical management.

Keywords: Congenital Abnormalities. Coronary artery disease. Sudden death

\section{Correspondencia:}

${ }^{*}$ Carlos A. Martínez-Cano

E-mail: karlosmartinezkano@ @otmail.com
Fecha de recepción: 17-02-2020

Fecha de aceptación: 05-08-2020

DOI: 10.24875/RCCAR.M22000125
Disponible en internet: 22-02-2022

Rev Colomb Cardiol. 2022;29(1):106-110

www.rccardiologia.com 0120-5633 / C 2022 Sociedad Colombiana de Cardiología y Cirugía Cardiovascular. Publicado por Permanyer. Este es un artículo open access bajo la licencia CC BY-NC-ND (http://creativecommons.org/licenses/by-nc-nd/4.0/). 


\section{Caso clínico}

Paciente de sexo femenino, de 42 años de edad, con antecedente de hipertensión arterial, en evaluación por médico del deporte, para quien solicitaron prueba de esfuerzo previa a recomendaciones de ejercicio, la cual fue reportada como máxima $88 \%$ negativa para isquemia, suspendida por fatiga a los 2 minutos y 30 segundos de la tercera etapa, logrando 9 MET. Durante la prueba de esfuerzo presentó un bloqueo completo de rama izquierda dependiente de frecuencia, que se resolvió al comienzo de la recuperación; no tuvo angina. Por la presencia de bloqueo de rama izquierda durante la prueba se decidió evaluar con angiografía por tomografía computarizada (angio-TC) de coronarias, en la que se evidenció un origen anómalo de la arteria descendente anterior (ADA), la cual se origina en el seno de Valsalva derecho, de manera independiente, haciendo un ángulo de $90^{\circ}$, para luego dirigirse a través del septum y entre el tracto de salida del ventrículo derecho y la aorta, al ventrículo izquierdo (sin trayecto intramural), sin definirse áreas de estenosis. Codominancia coronaria, fracción de eyección del $60 \%$ (Figs. 1-2). El electrocardiograma basal fue normal y la ecocardiografía transtorácica basal no mostró alteraciones; la fracción de eyección fue del $65 \%$.

Al reinterrogar a la paciente, refirió que desde su infancia notaba limitación para los esfuerzos extenuantes, fatiga con los mismos, sin disnea, angina, síncopes ni antecedentes familiares de muerte súbita 0 cardiopatías congénitas. El caso fue discutido con tres cirujanos cardiovasculares, dos de los cuales consideraron innecesaria una corrección quirúrgica; uno de ellos recomendó coronariografía para evaluar la compresión dinámica de la arteria durante el ciclo cardiaco, con miras a una posible intervención quirúrgica. Luego de discutir las opciones de manejo, la paciente decidió optar por el manejo médico; se agregó metoprolol al manejo antihipertensivo y se le recomendó abstenerse del ejercicio físico y de esfuerzos extenuantes, y asistir a seguimiento periódico por cardiología. Once meses después de tomar esta conducta, la paciente permanece asintomática, sin eventos cardiovasculares.

\section{Introducción}

El origen anómalo de las arterias coronarias es poco frecuente; fue descrito hace 2000 años por Galeno e ilustrado por primera vez por Vesalio en su Tabulea Anatomica. Actualmente, aun después de tanto tiempo de su conocimiento, su abordaje y su tratamiento siguen siendo un reto para los especialistas ${ }^{1}$.

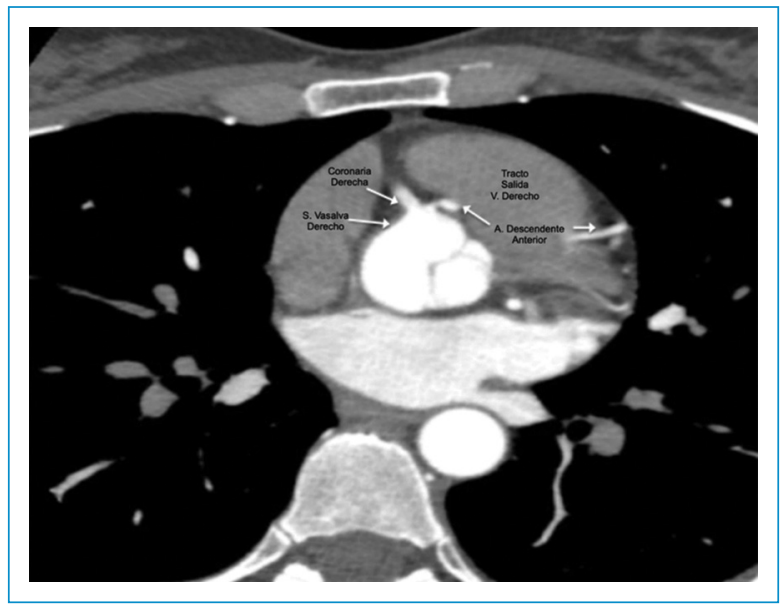

Figura 1. Corte axial en el que se aprecian el seno de Valsalva derecho, el origen de la coronaria derecha y la arteria descendente anterior, originándose de manera independiente y haciendo un ángulo de $90^{\circ}$ para dirigirse por el tracto de salida del ventrículo derecho hacia el ventrículo izquierdo, debajo de la válvula pulmonar.

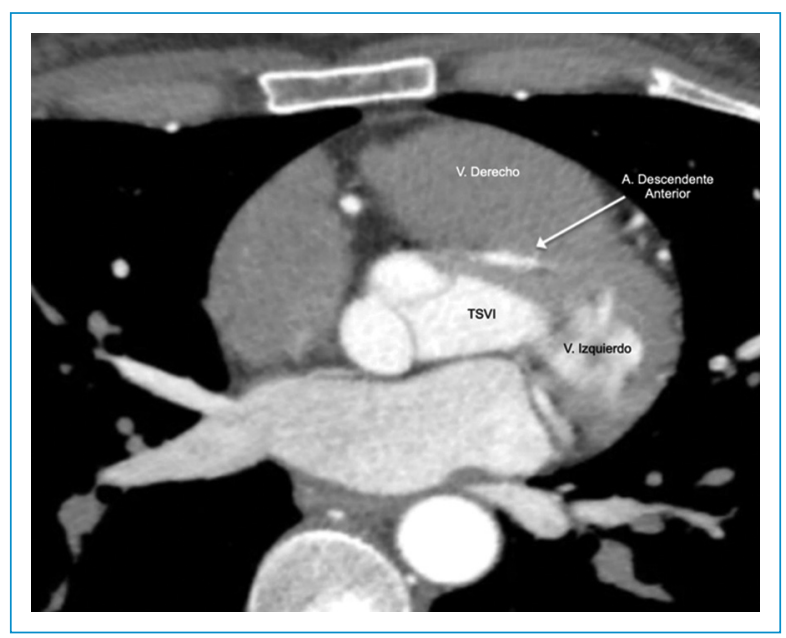

Figura 2. Corte axial en el que se aprecia el trayecto de la arteria descendente anterior por el septum interventricular hacia el ventrículo izquierdo. TSVI: tracto de salida del ventrículo izquierdo.

\section{Definición y epidemiología}

Se considera un origen anómalo de una arteria coronaria aquel que no tiene un origen típico desde su seno de Valsalva respectivo, implica un trayecto aberrante o presenta una fístula en el recorrido típico de la arteria $^{2}$. Se estima que un $80 \%$ de los pacientes serán asintomáticos en el transcurso de sus vidas y se diagnosticarán de manera incidental; el 20\% restante 
debutará con arritmias, síncope, infarto de miocardio o muerte súbita de causa cardiaca, convirtiéndose en la segunda causa más común de muerte súbita de causa cardiaca en jóvenes atletas ${ }^{3}$.

Se ha reportado una prevalencia de anomalías coronarias entre el $0.6 \%$ y el $1.3 \%$ en series de angiografía y del $0.3 \%$ en series de autopsias. Las más comunes son el origen independiente de la ADA y la circunfleja (Cx) en ausencia del tronco principal izquierdo (TPI), con un 64\%; la segunda en frecuencia es el origen anómalo de la coronaria derecha (CD) desde el seno de Valsalva izquierdo, con un $13 \%$, luego el origen de la Cx del seno de Valsalva derecho, con un $9 \%$, y finalmente el origen anómalo del TPI desde el seno de Valsalva derecho, con un $0.89 \%{ }^{4}$. Respecto al caso que se expone, con un origen de la ADA desde el seno de Valsalva derecho, se describen una incidencia del $0.008 \%$ y una prevalencia del $0.01 \%$ al $0,04 \%$, si bien juntos, origen coronario anómalo desde la arteria pulmonar, son anormalidades más infrecuentes ${ }^{4,5}$.

El origen anómalo de la ADA desde el seno de Valsalva derecho se presenta típicamente como angina estable o inestable, infarto de miocardio, arritmias ventriculares malignas y muerte súbita de causa cardiaca; son infrecuentes los casos asintomáticos. Se clasifica, según el trayecto de la ADA desde su origen en el seno de Valsalva derecho hasta el ventrículo izquierdo, en aquellos con curso por delante de la arteria pulmonar (prepulmonar), por detrás de la aorta (retroaórtica), por debajo de la válvula pulmonar (subvalvular pulmonar 0 transeptal) (Fig. 3) y entre la aorta y la arteria pulmonar (interarterial), también conocida como variante «maligna", dada su alta asociación con isquemia miocárdica y muerte súbita ${ }^{5-7}$. Además del curso interarterial de la $A D A$, hay otras características anatómicas de alto riesgo para eventos cardiacos, como son el ostium muy estrecho (slit-like), un ángulo de salida agudo menor de $45^{\circ}$, el curso intramural (dentro de la túnica media de la aorta) y un estrechamiento de más del $50 \%$ del vaso proximal comparado con el distal ${ }^{7,8}$. La evaluación de imagen multimodal es indispensable para definir las características anteriores.

\section{Diagnóstico}

En la actualidad, con el uso creciente de la imagen no invasiva para el diagnóstico cardiovascular, se ha logrado una mayor identificación de casos incidentales, por lo cual generan un gran reto el enfoque y el abordaje de estos pacientes ante la poca evidencia que existe sobre su tratamiento. A continuación, se presentan las ayudas diagnósticas más usadas para la detección y la evaluación del riesgo del origen anómalo de las arterias coronarias.

\section{Ecocardiografía}

La ecocardiografía transtorácica es un medio diagnóstico altamente disponible y de bajo costo, de alta utilidad en niños por la excelente ventana acústica, pero en adultos tiene un valor limitado ${ }^{9}$. Por su parte, la ecocardiografía transesofágica mejora la sensibilidad y la especificidad; sin embargo, se ha reportado una gran variabilidad interobservador, caso en el cual es determinante la experticia de cada centro. No se puede recomendar como estudio de elección frente a otras modalidades diagnósticas más precisas ${ }^{5,7}$.

\section{Angio-TC y resonancia magnética cardiaca}

Son las ayudas diagnósticas con mayor grado de recomendación. La angio-TC prevalece debido a la alta resolución espacial, la velocidad de adquisición y la amplia disponibilidad. Junto a la resonancia magnética (RM), permiten una adecuada visualización de las coronarias, su relación con estructuras adyacentes y la determinación de las características de alto riesgo ${ }^{7-9}$. La RM también permite evaluar la contractilidad miocárdica regional, la perfusión y la presencia de fibrosis, evitando la exposición a radiación y contraste yodado. Tiene la desventaja de necesitar mayor tiempo de adquisición y ofrecer un poco menor resolución espacial 7 . Estudios recientes sugieren la RM como un método de evaluación ideal que aporta gran información y puede ser realizado rápidamente, en 10 a 15 minutos, y sin mayores eventos adversos ${ }^{10,11}$.

\section{Angiografía y ultrasonido intravascular}

Originalmente, la mayoría de los hallazgos de anomalías coronarias se realizaban durante estudios angiográficos; sin embargo, la angiografía coronaria es una técnica invasiva que no permite una adecuada caracterización anatómica de estructuras adyacentes ni una visualización del ostium coronario y del trayecto arterial proximal. Algunos estudios muestran que hasta un $44 \%$ de los pacientes a quienes se realizó un estudio angiográfico necesitaron otro, como RM o TC. La principal utilidad de la angiografía está en la posibilidad de realizar ultrasonido intravascular (IVUS), que con su alta resolución espacial y temporal permite evaluar con 
precisión las características del ostium y el trayecto intramural, y puede valorar la dinámica vascular en condiciones de estrés con dobutamina o ejercicio. La tomografía por coherencia óptica aún no se ha estudiado mucho en este escenario $0^{5,7,12}$.

\section{Pruebas funcionales no invasivas}

Las pruebas de estrés con ejercicio son las más recomendadas, debido a que son más fisiológicas y asemejan las condiciones de ejercicio extremo en las cuales ocurren la mayoría de los casos de muerte súbita. Se puede realizar una prueba electrocardiográfica o imagenológica; no obstante, ambas pueden dar resultados falsos positivos y negativos. En un estudio de 27 atletas con origen anómalo de coronarias, seis de ellos tuvieron pruebas de esfuerzo negativas previo a un episodio de muerte súbita ${ }^{13}$. En otro estudio con prueba de perfusión miocárdica, se observó que la inducción de isquemia en esta población es muy rara ${ }^{14}$. Una prueba de estrés negativa no puede verse como tranquilizadora, especialmente cuando hay otros hallazgos anatómicos de alto riesgo o el paciente tiene síntomas ${ }^{5}$.

\section{Tratamiento}

El tratamiento de esta muy rara condición sigue siendo controvertido, como pudo verse en este caso, en el cual hubo discrepancia entre los conceptos de tres diferentes cirujanos cardiovasculares. En los más recientes consensos, las variantes de origen anómalo de la DA desde el ostium derecho, con curso que no es interarterial (subvalvular pulmonar o transeptal, retroaórtico, prepulmonar), se consideran benignas, y estos pacientes, en general, tienen un excelente pronóstico y usualmente no son referidos para cirugía, ni se les restringe el ejercicio, pero deben tener una prueba de esfuerzo previa antes de participar en deportes competitivos. Caso contrario son aquellos que, a pesar de tener estas variantes benignas, son sintomáticos, con angina o con inducción de isquemia demostrada o inducción de arritmias o muerte súbita abortada, en quienes está indicado el tratamiento quirúrgico ${ }^{12}$.

Los pacientes que tienen variantes interarteriales o malignas se consideran de peor pronóstico y se ha recomendado corrección quirúrgica a todos, incluso a quienes están asintomáticos ${ }^{12}$. Las guías americanas consideran la cirugía como una opción razonable para todos los pacientes con origen anómalo de la DA desde el seno de Valsalva izquierdo, aun sin curso interarterial y sin síntomas, con un grado de recomendación $11 a^{8}$.

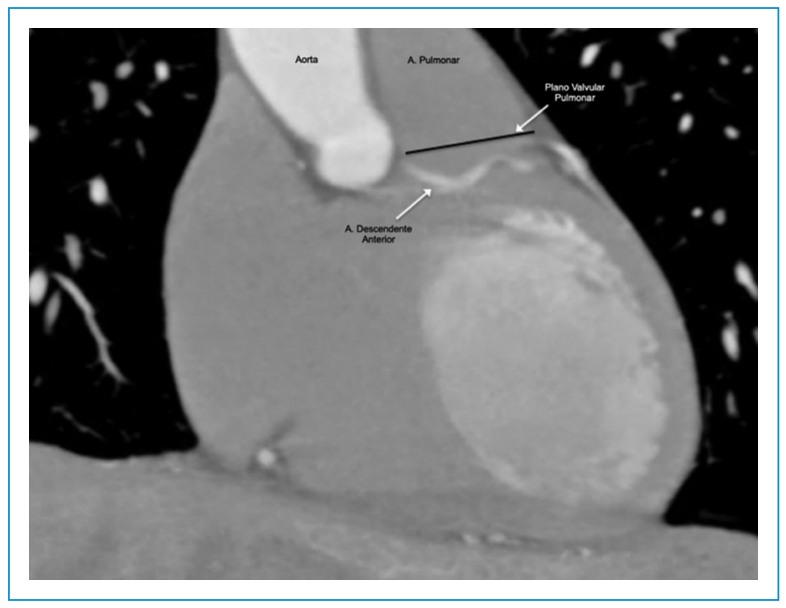

Figura 3. Corte coronal en el que se aprecia el curso subvalvular pulmonar, hacia el ventrículo izquierdo.

Otros grupos hacen énfasis en la evaluación de otras características de riesgo para eventos cardiovasculares, como ostium en hendidura, curso intramural, compresión coronaria y ángulo de salida agudo, como determinantes que hacen considerar la cirugía, incluso en pacientes asintomáticos ${ }^{15}$.

Los pacientes con origen de la CD desde el seno de Valsalva izquierdo se consideran una variante de bajo riesgo. En aquellos asintomáticos se considera la cirugía solo si se logra demostrar inducción de isquemia 0 arritmias cardiacas secundarias a isquemia. A aquellos con pruebas de ejercicio negativas puede permitírseles el ejercicio una vez discutidos, con el paciente y la familia, los potenciales riesgos, pues una prueba negativa para isquemia no excluye de manera definitiva la posibilidad de una muerte súbita ${ }^{8,12}$.

En algunos casos, como los pacientes que no aceptan la opción quirúrgica o los mayores de 35 años con variantes no interarteriales y sin otros determinantes de alto riesgo, como ocurre en el caso reportado, se ha descrito el manejo con beta-bloqueadores y se han reportado buenos desenlaces a largo plazo ${ }^{12,15}$.

\section{Conclusiones}

El origen anómalo de las arterias coronarias es una anormalidad congénita bastante rara. El origen anómalo de la DA desde el seno de Valsalva derecho es la presentación más rara, solo superada por el origen anómalo desde la arteria pulmonar. La presentación clínica va desde angina hasta muerte súbita. Las anomalías coronarias son la segunda causa de muerte súbita en atletas 
jóvenes. El diagnóstico y la evaluación requieren un abordaje multimodal, pues no todos los pacientes precisan corrección quirúrgica. La decisión de tratamiento implica una evaluación clínica juiciosa, imágenes y funcionalidad, y la discusión idealmente en centros expertos entre los cardiólogos clínicos y cirujanos cardiovasculares, para optar por el tratamiento más adecuado.

\section{Financiamiento}

Los autores declaran no haber recibido
financiamiento.

\section{Conflicto de intereses}

Los autores declaran no tener conflicto de intereses.

\section{Responsabilidades éticas}

Protección de personas y animales. Los autores declaran que para esta investigación no se han realizado experimentos en seres humanos ni en animales.

Confidencialidad de los datos. Los autores declaran que han seguido los protocolos de su centro de trabajo sobre la publicación de datos de pacientes.

Derecho a la privacidad y consentimiento informado. Los autores han obtenido el consentimiento informado de los pacientes y/o sujetos referidos en el artículo. Este documento obra en poder del autor de correspondencia.

\section{Bibliografía}

1. Mosca RS, Phoon CK. Anomalous aortic origin of a coronary artery is not always a surgical disease. Semin Thorac Cardiovasc Surg Pediatr Card Surg Annu. 2016;19:30-6.

2. Lluri G, Aboulhosn J. Coronary arterial development: a review of normal and congenitally anomalous patterns. Clin Cardiol. 2014;37:126-30.

3. Graidis C, Dimitriadis D, Karasavvidis V, Dimitriadis G, Argyropoulou E, Economou $F$, et al. Prevalence and characteristics of coronary artery anomalies in an adult population undergoing multidetector-row computed tomography for the evaluation of coronary artery disease. BMC Cardiovasc Disord. 2015;15:112.

4. Yildiz A, Okcun B, Peker T, Arslan C, Olcay A, Bulent Vatan M. Prevalence of coronary artery anomalies in 12,457 adult patients who underwent coronary angiography. Clin Cardiol. 2010;33:E60-4.

5. Cheezum MK, Liberthson RR, Shah NR, Villines TC, O'Gara PT, Landzberg MJ, et al. Anomalous aortic origin of a coronary artery from the inappropriate sinus of Valsalva. J Am Coll Cardiol. 2017;69:1592-608.

6. Tuncer C, Batyraliev T, Yilmaz R, Gokce M, Eryonucu B, Koroglu S. Origin and distribution anomalies of the left anterior descending artery in 70,850 adult patients: multicenter data collection. Catheter Cardiovasc Interv. 2006;68:574-85.

7. Grani C, Buechel RR, Kaufmann PA, Kwong RY. Multimodality imaging in individuals with anomalous coronary arteries. JACC Cardiovasc Imaging. 2017;10:471-81.

8. Stout KK, Daniels CJ, Aboulhosn JA, Bozkurt B, Broberg CS, Colman JM, et al. 2018 AHA/ACC Guideline for the management of adults with congenital heart disease: a report of the American College of Cardiology/ American Heart Association Task Force on Clinical Practice Guidelines. Circulation. 2019;139:e698-e800.

9. Angelini P. Imaging approaches for coronary artery anomalies: purpose and techniques. Curr Cardiol Rep. 2019;21:101.

10. Angelini $P$, Cheong BY, Lenge De Rosen VV, López A, Uribe C Masso $\mathrm{AH}$, et al. High-risk cardiovascular conditions in sports-related sudden death: prevalence in 5,169 schoolchildren screened via cardiac magnetic resonance. Tex Heart Inst J. 2018;45:205-13.

11. Prakken NH, Cramer MJ, Olimulder MA, Agostoni $P$, Mali WP, Velthuis BK. Screening for proximal coronary artery anomalies with 3-dimensional MR coronary angiography. Int J Cardiovasc Imaging. 2010;26:701-10.

12. Brothers JA, Frommelt MA, Jaquiss RDB, Myerburg RJ, Fraser CD Jr, Tweddell JS. Expert consensus guidelines: anomalous aortic origin of a coronary artery. J Thorac Cardiovasc Surg. 2017;153:1440-57.

13. Basso C, Maron BJ, Corrado D, Thiene G. Clinical profile of congenital coronary artery anomalies with origin from the wrong aortic sinus leading to sudden death in young competitive athletes. J Am Coll Cardiol. 2000;35:1493-501.

14. Grani C, Benz DC, Schmied C, Vontobel J, Mikulicic F, Possner M, et al. Hybrid CCTA/SPECT myocardial perfusion imaging findings in patients with anomalous origin of coronary arteries from the opposite sinus and suspected concomitant coronary artery disease. J Nucl Cardiol. 2017;24:226-34.

15. Molossi S, Martínez-Bravo LE, Mery CM. Anomalous aortic origin of a coronary artery. Methodist Debakey Cardiovasc J. 2019;15:111-21. 